\section{Asociación entre síntomas depresivos y consumo abusivo de alcohol en estudiantes de Bucaramanga, Colombia}

\author{
Association between depressive symptoms \\ and alcohol abuse among students \\ from Bucaramanga, Colombia
}

\author{
Diana Carolina Rodríguez Rodríguez 1 \\ Carlos Manuel Dallos Bareño 1 \\ Silvia Juliana González Rueda 1 \\ Zayda Marcela Sánchez Herrera 1 \\ Luis Alfonso Díaz-Martínez 1 \\ Germán Eduardo Rueda-Jaimes 1 \\ Adalberto Campo-Arias 1
}

\footnotetext{
1 Facultad de Medicina, Universidad Autónoma de Bucaramanga, Bucaramanga, Colombia.

Correspondencia A.Campo-Arias Facultad de Medicina, Universidad Autónoma de Bucaramanga. Calle 157 \# 19-55, Cañaveral Parque, Bucaramanga, Colombia. acampoar@unab.edu.co
}

\begin{abstract}
Various studies have reported an important association between depressive symptoms and alcohol abuse. However, the topic had not been investigated in Colombian students. This study focused on the association between depressive symptoms and alcohol abuse among high school students in Bucaramanga, Colombia. A self-reported anonymous questionnaire was answered by 560 15-19-year-olds. The survey included the Zung Self-Reported Depression Scale, CAGE Questionnaire for Alcohol Use, and VESPA questionnaire (Epidemiological Surveillance of Psychoactive Drugs). Logistic regression was used to establish associations. Prevalence was 5.7\% for alcohol dependence and 39.5\% for depressive symptoms. Associations were found between alcohol abuse and depressive symptoms $(P R=3.33$; 95\%CI: 1.41-7.83), poor self-perceived academic achievement $(P R=2.59 ; 95 \% C I$ : 1.16-5.37), and smoking ( $P R=2.47$; 95\%CI: 1.13-5.40). The authors conclude that there is a strong association between depressive symptoms and alcohol abuse in Colombian high school students. Preventive programs are needed to identify early depressive disorders and alcohol abuse.
\end{abstract}

Alcohol Drinking; Depression; Adolescent

\section{Introducción}

En Colombia el alcohol en todas las edades es la sustancia más utilizada de aquellas que pueden causar abuso o inducir dependencia 1,2. El uso de bebidas alcohólicas es frecuente por influencia y aceptación social de su consumo ${ }^{3}$. En consecuencia, el consumo de alcohol es frecuente en adolescentes y está relacionado con diversos factores individuales y del contexto social 4 . Se ha observado una asociación significativa entre el consumo de alcohol con otros comportamientos de riesgo para la salud. El consumo de alcohol se encuentra con más frecuencia en adolescentes que consumen otras sustancias susceptibles de abuso, que realizan poca actividad física y muestran conductas sexuales de riesgo 5 . De la misma forma, el consumo frecuente de alcohol, en un número considerable de adolescentes, está asociado a la presencia de síntomas depresivos 4,6. Se ha sugerido que el consumo abusivo de alcohol es un síntoma de un trastorno depresivo no identificado 7 . Es posible que el alcohol sea utilizado como una forma de afrontamiento del estado de ánimo disfórico 8,9.

Desde el punto de vista de salud pública, es muy importante identificar la relación existente entre síntomas depresivos y el consumo abusivo de alcohol. Si los síntomas depresivos predicen el inicio del consumo de alcohol, es posible que la identificación temprana y el manejo 
oportuno de síntomas depresivos con importancia clínica prevengan el consumo abusivo de alcohol 10,11. De igual manera, se prevendría el consumo de otras sustancias susceptibles de abuso o causa de dependencia. Se acepta que un número relevante de consumidores de sustancias se inician con la ingesta de alcohol 12 .

El objetivo de este estudio fue determinar la asociación entre el consumo abusivo de alcohol y síntomas depresivos, ajustando por sexo, consumo de cigarrillos, percepción de rendimiento académico y percepción del estado de salud, en estudiantes de educación media vocacional (décimo y undécimo grado) de $\mathrm{Bu}$ caramanga, Colombia.

\section{Método}

El presente es un estudio de corte transversal. Este trabajo es producto del proceso de investigación formativa realizado en la Universidad Autónoma de Bucaramanga y fue aprobado por el Centro de Investigaciones y el Comité de Ética de la Universidad. Todos los participantes lo hicieron de forma voluntaria y firmaron un consentimiento informado.

Por conveniencia se tomaron cuatro colegios de Bucaramanga, Colombia, dos públicos y dos privados que fueran mixtos. En el momento de la investigación, primer semestre del 2003, había 2.623 estudiantes cursando décimo y undécimo grado, media vocacional. Se calculó una muestra representativa, estratificada por colegio, con un nivel de confianza del $95 \%$ y un poder del $80 \%$. La prevalencia esperada para síntomas depresivos con importancia clínica fue $25,0 \%$, una frecuencia de consumo abusivo de alcohol de 5,8\% entre los estudiantes sin síntomas depresivos con importancia clínica y de 14,5\% (2,5 más alta) más en los estudiantes con síntomas depresivos clínicamente relevantes. La muestra final fue de 600 estudiantes. Se incluyeron aquellos estudiantes con edad comprendida entre 15 y 19 años. Todos los estudiantes fueron seleccionados al azar por un muestreo aleatorio simple.

Para investigar consumo de sustancias se utilizó una versión modificada del formulario 1 del Sistema de Vigilancia Epidemiológica para el Uso Indebido de Sustancias Psicoactivas (VESPA) de amplio uso en Colombia. Este formulario autoadministrado indaga sobre consumo de cigarrillos, cannabis, bazuco, cocaína, alcohol y otras sustancias en el encuestado y en amigos cercanos y familiares. Además, investiga sobre la percepción subjetiva de salud y de rendimiento académico durante el mes anterior 13. El formulario fue diligenciado en el aula de clase y bajo supervisión para aclarar las dudas que se pudieran presentar.

Los síntomas depresivos durante las dos últimas semanas fueron cuantificados usando la escala para depresión de Zung. Este instrumento tipo Likert consta de veinte preguntas con cuatro opciones de respuestas que se responde nunca, a veces, algunas veces y casi siempre. Da un puntaje de uno a cuatro a cada respuesta. La puntuación total oscila entre 20 y 80 . Este resultado debe ser multiplicado por el factor 1,25 , para obtener puntuaciones finales entre 25 y 100 puntos. Valores iguales o superiores a 50 sugieren síntomas depresivos de importancia clínica 14 . No se cuenta con validación formal de esta escala en población adolescente; no obstante, ha mostrado una buena consistencia interna (un valor de alfa de Cronbach 0,80 ) en adolescentes estudiantes de una ciudad colombiana 15 .

Para identificar el consumo abusivo de alcohol se utilizó la escala CAGE. Este instrumento es un instrumento de tamizaje que incluye cuatro preguntas relacionadas con la culpabilidad individual, la crítica social, la necesidad de disminuir el consumo y la ingestión matutina. A cada respuesta afirmativa se da un punto. Puntuaciones de tres o cuatro sugieren un consumo abusivo de alcohol 16. La escala CAGE no ha sido validada de manera formal en Colombia, sin embargo, es la escala más ampliamente utilizada por su brevedad y por mostrar una aceptable sensibilidad y especificidad $(0,86$ 0,90 y $0,78-0,81$ ) cuando de toma dos o más como punto de corte que sugiere problemas con el consumo de alcohol 17.

Los datos fueron almacenados y procesados en SPSS 11.0. Se realizó el análisis univariado para calcular frecuencias, porcentajes (con intervalos de confianza del 95\%, IC95\%), promedios y desviación estándar (DE). El análisis bivariado se realizó para calcular razones de prevalencia (RP) con IC95\%. El análisis multivariado se realizó mediante un modelo de regresión logística se establecieron odds ratios (OR) con IC95\% y bondad de ajuste de HosmerLemeshow 18. Se incluyeron como variables de confusión las variables que en el análisis bivariado alcanzaron valores de probabilidad menores del 25,0\%, lo mismo que el sexo. Se aceptaron como significativos valores de $\mathrm{p}<0,05$. 
Esta investigación siguió los principios éticos para la investigación con seres humanos contenidos en la Declaración de Helsinki y las normas vigentes para la investigación con seres humanos en Colombia que califica este tipo de investigación como de riesgo mínimo para la integridad física y mental de los participantes 19 .

\section{Resultados}

De la muestra calculada de 600 estudiantes, $560(93,3 \%)$ fueron incluidos en este informe. Se excluyeron 40 estudiantes, 25 por ser menores de 15 años de edad y 15 que se negaron a participar.

La edad promedio del grupo fue de 15,8 años ( $\mathrm{DE}=0,89$ ), la moda de 15 años, la mediana de 16 años y el 75,0\% de los encuestados tenía 15 ó 16 años de edad. En relación con el sexo, 315 (56,3\%) eran varones y 245 (43,8\%) eran mujeres. En lo concerniente al grado de escolaridad cursado, $288(51,4 \%)$ estudiantes cursaban décimo grado y $272(48,6 \%)$ undécimo grado. Respecto a la percepción de rendimiento, $369(65,9 \%)$ estudiantes manifestaron tener entre excelente y buen rendimiento y $191(34,1 \%)$ estudiantes de regular a malo. En cuanto a la percepción de salud, $463(82,7 \%)$ estudiantes informaron un estado excelente o bueno y 97 $(17,3 \%)$ regular o malo. En cuanto al consumo sustancias, 97 (17,3\%) encuestados manifestaron consumo actual de cigarrillos, 11 (2,0\%) consumo de cannabis durante el último año y 327 (58,4\%; IC95\%: 54,2-62,6) consumo de alcohol durante el último año, de los cuales 5,7\% (IC95\%: 3,7-7,7) mostró un consumo abusivo de alcohol. En relación con el consumo de sustancias en personas conocidas, 267 (47,7\%) estudiantes informaron que el mejor amigo acos- tumbraba consumir alcohol o cigarrillos y 355 $(63,4 \%)$ afirmaron que los familiares cercanos consumían sustancias como alcohol o cigarrillos. Por su parte, las puntuaciones en la escala de Zung oscilaron entre 26 y 94 puntos, con una media de 47,8 $(\mathrm{DE}=9,9)$. Un total de 221 (39,5\%; IC95\%: 35,4-43,6) estudiantes alcanzaron puntuaciones de síntomas depresivos con importancia clínica, 50 o más puntos. Los síntomas depresivos eran leves en 157 (28,0\%), moderados en $49(8,8 \%)$ y severos en $15(2,7 \%)$.

En el análisis bivariado se observó que el consumo abusivo de alcohol se encontraba con mayor frecuencia en quienes informaron una percepción de salud regular o mala $(11,3 \%$ frente a $4,5 \%$; RP $=2,50$; IC95\%: $1,25-5,01)$, rendimiento académico regular o malo (10,5\% frente a $3,3 \%$; RP $=3,22$; IC95\%: $1,61-6,45)$, consumo actual de cigarrillos $(13,4 \%$ frente a $4,1 \%$; $\mathrm{RP}=3,27$; IC95\%: 1,67-6,39), el consumo de alcohol o cigarrillos por el mejor amigo $(8,2 \%$ frente a $3,4 \%$; RP $=2,41$; IC95\%: $1,16-5,00$ ), el consumo de alcohol o cigarrillos por familiares $(7,6 \%$ frente a $2,4 \% ; \mathrm{RP}=3,12$; IC95\%: $1,22-7,97)$ y la presencia de síntomas depresivos con importancia clínica $(10,4 \%$ frente a $2,7 \%$; $\mathrm{RP}=3,97$; IC95\%: 1,85-8,31). Ver Tabla 1. El consumo abusivo de alcohol fue independiente de la edad, el sexo y el grado de escolaridad.

En el análisis multivariado se mantuvo la asociación significativa del consumo abusivo de alcohol con la presencia de síntomas depresivos, la sensación de mal o regular rendimiento académico y el consumo de cigarrillos. En este modelo se incluyó el sexo como covariable; aunque en el análisis bivariado mostró un valor de probabilidad mayor del $25,0 \%$ para ajustar el posible efecto de confusión entre síntomas depresivos y consumo abusivo de alcohol. Este modelo ajustaba adecuadamente $(p=0,62)$. Ver Tabla 2. En este análisis la asociación entre

Tabla 1

Asociación entre la severidad de los síntomas depresivos y consumo abusivo de alcohol en 560 estudiantes de medio vocacional de Bucaramanga, Colombia.

\begin{tabular}{|c|c|c|c|c|c|c|}
\hline \multirow[t]{2}{*}{ Variable } & \multicolumn{2}{|c|}{$\begin{array}{l}\text { CAGE positivo } \\
(n=32)\end{array}$} & \multicolumn{2}{|c|}{$\begin{array}{c}\text { CAGE negativo } \\
(n=528)\end{array}$} & \multirow[t]{2}{*}{$\mathbf{R P}$} & \multirow[t]{2}{*}{ IC95\% } \\
\hline & $\mathrm{n}$ & $\%$ & $\mathrm{n}$ & $\%$ & & \\
\hline Sin síntomas depresivos & 9 & 28,1 & 330 & 62,5 & 1,00 & Referencia \\
\hline Síntomas leves & 14 & 43,8 & 143 & 27,1 & 3,36 & $1,49-7,59$ \\
\hline Síntomas moderados & 7 & 21,9 & 42 & 8,0 & 5,38 & $2,10-13,79$ \\
\hline Síntomas severos & 2 & 6,2 & 13 & 2,4 & 5,02 & $1,19-21,25$ \\
\hline
\end{tabular}

$\mathrm{RP}=$ razón de prevalencia; $\mathrm{IC95 \%} \mathrm{=} \mathrm{intervalo} \mathrm{de} \mathrm{confianza} \mathrm{del} \mathrm{95 \% .}$ 
la severidad de los síntomas depresivos y el consumo abusivo de alcohol perdió significancia estadística.

\section{Discusión}

En este estudio se encuentra un consumo abusivo de alcohol en aproximadamente uno de cada veinte estudiantes de enseñanza media vocacional. La presencia de síntomas depresivos con importancia clínica y el consumo abusivo de alcohol mantienen su asociación, aún después de ajustar por sexo, consumo de cigarrillos y un rendimiento académico inferior.

La prevalencia de consumo de alcohol varía de una región a otra y en los diferentes grupos de edad. Los resultados de este estudio muestran una prevalencia de consumo abusivo de alcohol en el 5,7\%. La prevalencia de consumo abusivo de alcohol varía según el método utilizado en la evaluación del uso de alcohol. En 1997, Rueda 20 informó un consumo abusivo de alcohol del 5,8\% en estudiantes de educación media vocacional de Bucaramanga mediante el uso, igualmente, del cuestionario CAGE. Mientras que en otros países se han informado prevalencias superiores de consumo de alcohol, por ejemplo, Bailey 21 halló una frecuencia del $26,4 \%$ de bebedores habituales; en tanto que Walter et al. 22 encontraron que 15,5\% informó una alta ingesta de bebidas alcohólicas en estudiantes de enseñanza media vocacional (high school).

En relación con el sexo, en adolescentes durante los últimos años el consumo de alcohol presenta un patrón similar en varones y en mujeres 21,23 . Se ha observado un aumento gradual en el consumo de alcohol por las mujeres, alcanzando valores similares a los observados en los varones, y esta tendencia parece comenzar desde la adolescencia 24. En nuestro estudio encontramos que el consumo abusivo de alcohol era independientemente del sexo, hallazgo similar al informado por Torres et al. 13 que usaron el cuestionario CAGE.

Por otra parte, en el análisis multivariado se encontró que los estudiantes con consumo abusivo de alcohol informaron estado de salud comparable a los no consumidores. Este hallazgo es inconsistente con otros estudios, Torres et al. 13 y Rueda \& Camacho 25 informaron que el consumo de alcohol y otras sustancias, con excepción del consumo de cigarrillos, se asocia a una percepción menos positiva del estado de salud. Es posible, que esta diferencia pueda ser explicada porque en estas investigaciones no se realizó un análisis multivariado
Tabla 2

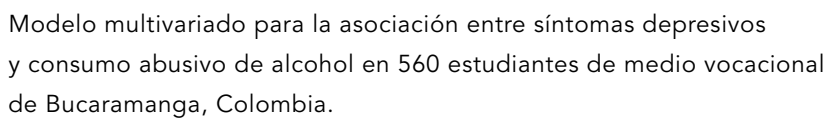

\begin{tabular}{lcc}
\hline CAGE & OR* & IC95\% \\
\hline Síntomas depresivos con importancia clínica & 3,33 & $1,41-7,83$ \\
Sexo masculino & 1,59 & $0,71-3,55$ \\
Fumar cigarrillos & 2,47 & $1,13-5,40$ \\
Rendimiento académico regular o malo & 2,50 & $1,16-5,37$ \\
Percepción de salud regular o mala & 1,73 & $0,75-3,98$ \\
\hline
\end{tabular}

OR = odds ratio; IC $95 \%$ = intervalo de confianza del 95\%.

* Ajustado por las otras variables.

para controlar la confusión que implican otras variables 26 . Se acepta que las lesiones en órganos específicos guardan relación significativa con el tiempo en que se ha consumido de alcohol, los efectos negativos sobre la salud se observan con mayor frecuencia después de varios años de consumo frecuente 3 .

Los hallazgos de estudio son consistentes con estudios precedentes que informan una asociación significativa entre el consumo de cigarrillos y el consumo abusivo de alcohol en adolescentes 21,27,28. En la actualidad, hay suficiente evidencia que indica que las conductas de riesgo para la salud forman parte de un patrón de comportamiento que se encuentra en el mismo grupo de adolescentes 5,29,30.

De la misma forma, los estudiantes con consumo abusivo de alcohol informaron una mayor proporción de rendimiento académico regular o malo durante el último mes. Hallazgo similar informaron Pérez \& Pinzón-Pérez 31, quienes encontraron que los estudiantes bogotanos que habían tomado alcohol durante los días previos percibían de manera inferior sus habilidades académicas. A su vez, Katja et al. 32 encontraron en estudiantes finlandeses que la satisfacción en el colegio se relacionaba con un bajo consumo de alcohol. El tipo de diseño transversal de nuestra investigación no permite establecer el sentido de la asociación, pero es posible una relación bidireccional 33 .

La asociación encontrada en este estudio entre síntomas depresivos y consumo abusivo de alcohol es concordante con los hallazgos de otros estudios 34,35. De la misma forma, los datos muestran que el consumo de alcohol se incrementa con la severidad de los síntomas depresivos. Kelder et al. 36 observaron que la asociación era mayor a medida que se incrementaba el número de síntomas depresivos, la rela- 
ción era más significativa cuando se encontraban frente a un episodio depresivo mayor.

El consumo abusivo de alcohol es un problema de salud pública relacionado con múltiples causas. Estudios recientes informan el papel que juegan los factores hereditarios en interacción con factores medioambientales 37,38,39,40. Sin embargo, algunos datos sugieren que el entorno sociocultural es tan determinante como el componente genético 22,41,42.

Sin duda, la relación existente entre consumo abusivo de alcohol y el consumo de otras sustancias es de capital importancia. Es probable, que la prevención del consumo abusivo de alcohol disminuya el inicio de consumo de otras sustancias como nicotina (cigarrillo), cannabis

\section{Resumen}

Existe una asociación significativa entre síntomas depresivos y consumo abusivo de alcohol en adolescentes; sin embargo, ésta no ha sido investigada en estudiantes colombianos. El objetivo de este estudio fue establecer la asociación entre síntomas depresivos y consumo abusivo de alcohol en estudiantes de educación media vocacional de Bucaramanga, Colombia. Una encuesta anónima que incluía la escala para depresión de Zung, la escala para alcoholismo de CAGE y el cuestionario VESPA fue diligenciada por una muestra aleatoria de 560 estudiantes entre 15 y 19 años de edad. La fuerza de asociación se estableció con un modelo de regresión logística. La prevalencia de síntomas depresivos con importancia clínica fue 39,5\% y la prevalencia de consumo abusivo de alcohol fue 5,7\%. Se encontró una asociación entre síntomas depresivos ( $R P=3,33$; IC95\%: 1,41-7,83), percepción de pobre rendimiento académico $(R P=2,50$; IC95\%: 1,16-5,37) y fumar cigarrillos $(R P=2,47$; IC95\%: 1,13-5,40) con consumo abusivo de alcohol. Se concluye que existe una asociación importante entre síntomas depresivos con importancia clínica y consumo abusivo de alcohol. Es necesaria la identificación temprana de depresión y el consumo abusivo de alcohol en adolescentes.

Consumo de Bebidas Alcohólicas; Depresión; Adolescente

\section{Colaboradores}

D. C. R. Rodríguez, C. M. D. Bareño, S. J. G. y R. Z. M. S. Herrera participaron en el diseño, la recolección de los datos y el análisis estadístico y la elaboración de este informe. L. A. Díaz-Martínez y A. Campo-Arias contribuyeron en el diseño, análisis estadístico y en la elaboración de este informe. G. E. Rueda-Jaimes colaboró en el diseño y la elaboración de este informe. o cocaína, basado en que existe una asociación importante entre el consumo de alcohol y otras sustancias en adolescentes estudiantes 21 . Asimismo, la identificación de síntomas depresivos con importancia clínica puede reducir el inicio de consumo de alcohol. Se ha observado que los síntomas depresivos son un factor mediador importante para el inicio de consumo de sustancias en individuos vulnerables 43,44,45.

Se concluye que existe una asociación importante entre el informe de síntomas depresivos y el consumo abusivo de alcohol en adolescentes estudiantes. Es necesario implementar programas preventivos que reduzcan el consumo abusivo de alcohol y de identificación de casos de depresión.

\section{Referências}

1. Rodríguez E. Estudio nacional sobre consumo de sustancias psicoactivas en Colombia 1996. Bogotá: Editorial Carrera 7a Ltda; 1997.

2. Posada JA, Torres Y. Estudio nacional de salud mental y consumo de sustancias psicoactivas, Colombia, 1993. Bogotá: Ministerio de Salud de Colombia; 1995.

3. Velásquez E. Alcoholismo. In: Toro RJ, Yepes LE, editores. Fundamentos de medicina, psiquiatría. 3a Ed. Medellín: Corporación para Investigaciones Biológicas; 1997. p. 67-80.

4. Bailey GW. Children, adolescents, and substance abuse. J Am Acad Child Adolesc Psychiatry 1992; 31:1015-8.

5. Leigh BC, Morrison DM. Alcohol consumption and sexual risk-taking in adolescents. Alcohol Health Res World 1991; 15:58-63.

6. Gleid S, Pine D. Consequences and correlates of adolescent depression. Arch Pediatr Adolesc Med 2002; 156:1009-14.

7. DeMilio L. Psychiatric syndromes in adolescent substance abusers. Am J Psychiatry 1989; 146:1212-4.

8. Mora-Ríos J, Natera G. Expectativa, consumo de alcohol y problemas asociados en estudiantes universitarios de la ciudad de México. Salud Pública Méx 2001; 43:89-96.

9. Bukstein OG, Brent DA, Kaminer Y. Comorbidity of substance abuse and other psychiatric disorders in adolescents. Am J Psychiatry 1989; 146: 1131-41.

10. Mora-Medina ME, Craviotto P, Villatoro J, Fleiz C, Galván-Castillo F, Tapia-Conyer R. Consumo de drogas entre adolescentes: resultados de la Encuesta Nacional de Adicciones, 1998. Salud Pública Méx. 2003; 45 Suppl 1:S16-25.

11. Hill S, Locke J, Lowers L, Connolly J. Psychopathology and achievement in children at high risk for developing alcoholism. J Am Acad Child Adolesc Psychiatry 1999; 38:883-91.

12. Brook DW, Brook JS, Zhang C, Cohen P, Whiteman M. Drug use and the risk of major depressive disorder, alcohol dependence, and substance use disorders. Arch Gen Psychiatry 2002; 59:1039-44. 
13. Torres Y, Muñoz AL, Ramírez H. Vigilancia epidemiológica sobre el uso indebido de sustancias psicoactivas “VESPA” Medellín, Antioquia 1992. Bol Epidemiol Antioq 1994; XIX:180-93.

14. Zung WWK. Self-report depression scale. Arch Gen Psychiatry 1965; 12:63-70.

15. De La Cruz D, Mariano L. Prevalencia de síntomas depresivos en estudiantes de 11ㅇ del Colegio Comfenalco de Cartagena. Cartagena: Universidad Tecnológica de Cartagena; 2000.

16. Ewing JA. Detecting alcoholism. The CAGE questionnaire. JAMA 1984; 252:1905-7.

17. Ewing JA. CAGE questionnaire. In: Rush AJ, Pincus HA, First MB, Zarin DA, Blacker D, Endicot J, et al., editors. Handbook of psychiatric measures [CD-ROM]. Washington DC: American Psychiatric Association; 2002.

18. Hosmer DW, Taber S, Lemeshow S. The importance of assessing the fit of logistic regression models: a case study. Am J Public Health 1991; 81:1630-5.

19. Ministerio de Salud de Colombia. Resolución 008430 de 1993 , por la cual se establecen las normas científicas, técnicas y administrativas para la investigación en salud. Bogotá: Ministerio de Salud de Colombia; 1993.

20. Rueda GE. Diagnóstico del consumo de sustancias psicoactivas en los estudiantes de enseñanza media vocacional de Bucaramanga y su área metropolitana en 1996. Bucaramanga: Grafisur; 1997.

21. Bailey SL. Adolescent's multisubstance use pattern: the role of heavy alcohol and cigarette use. Am J Public Health 1992, 82:1220-4.

22. Walter HJ, Vaughan RD, Cohall AT. Risk factors for substance use among high school students: implications for prevention. J Am Acad Child Adolesc Psychiatry 1991; 30:556-62.

23. Sutherland I, Willner P. Patterns of alcohol, cigarette and illicit drug use in English adolescents. Addiction 1998; 93:1199-208.

24. Kendler KS, Heath AC, Neale MC, Kessler RC, Eaves LJ. A population-based twin study of alcoholism in women. JAMA 1992; 268:1877-82.

25. Rueda GE, Camacho PA. Vigilancia epidemiológica sobre el uso indebido de sustancias psicoactivas en los estudiantes de enseñanza media vocacional de Bucaramanga y su área metropolitana. Médicas UIS 1998; XII:107-13.

26. Rodríguez MN, Ruiz JG. Plan de análisis. In: Ruiz A, Gómez C, Londoño D, editores. Investigación clínica: epidemiología clínica aplicada. Bogotá: Centro Editorial Javeriano; 2001. p. 491-502.

27. Poikolainen K, Tuulio-Henkiksson A, Aalto-Setälä T, Marttunen M, Lönnqvist J. Predictor of alcohol intake and heavy drinking in early adulthood: a 5-year follow-up of 15-19-year-old Finnish adolescents. Alcohol Alcohol 2001; 36:85-8.

28. Eckhardt L, Woodruff SI, Elder JP. A longitudinal analysis of adolescent smoking and its correlates. J Sch Health 1994; 64:67-72.

29. Atav S, Spencer GA. Health risk behaviors among adolescents attending rural, suburban, and urban schools: a comparative study. Fam Community Health 2002; 25:53-64.

30. Everett SA, Malarcher AM, Sharp DJ, Husten CG, Giovino GA. Relationship between cigarette, smoke- less tobacco, and cigar use, and other health risk behaviors among U.S. high school students. J Sch Health 2000; 70:234-40.

31. Pérez MA, Pinzón-Pérez H. Alcohol, tobacco, and other psychoactive drug use among high school students in Bogotá, Colombia. J Sch Health 2000; 70:377-80.

32. Katja R, Päivi A-K, Marja-Terttu T, Pekka L. Relationship among adolescent subjective well-being, health behavior, and school satisfaction. J Sch Health 2002; 72:243-9.

33. Cedeño M, Pardo G. Investigación descriptiva. In: Pardo G, Cedeño M, editors. Investigación en salud. Factores sociales. Bogotá: McGraw-Hill Interamericana; 1997. p. 118-33.

34. Alva SA. Psychological distress and alcohol use in Hispanic adolescents. J Youth Adolesc 1995; 24: 481-97.

35. Scheier LM, Botvin GJ, Baker E. Risk and protective factors as predictors of adolescents alcohol involvement and transition in alcohol use: a prospective analysis. J Stud Alcohol 1997; 58:652-67.

36. Kelder SH, Murria NG, Orpinas P, Prokhorov A, McReynolds L, Zhang Q, et al. Depression and substance use in minority middle-school students. Am J Public Health 2001; 91:761-6.

37. Fu Q, Heath AC, Bucholz KK, Nelson E, Goldberg J, Lyons MJ, et al. Shared genetic risk of major depression, alcohol dependence, and marijuana dependence. Contribution of antisocial personality disorder in men. Arch Gen Psychiatry 2002; 59: 1125-32.

38. Boyle MH, Sanford M, Szatmari P, Merikangas K, Offord DR. Familial influences on substance use by adolescents and young adults. Can J Public Health 2001; 92:206-9.

39. Han C, McGue MK, Iacono WG. Lifetime tobacco, alcohol and other substance use in adolescent Minnesota twins: univariate and multivariate behavioral genetic analyses. Addiction 1999; 94:981-93.

40. Lowry R, Kann L, Collins JL, Kolbe LJ. The effect of socioeconomic status on chronic disease risk behaviors among US adolescents. JAMA 1996; 276:792-7.

41. Griffin KW, Scheier LM, Botvin GJ, Diaz T. Ethnic and gender differences in psychosocial risk, protection, and adolescent alcohol use. Prev Sci 2000; 1:199-212.

42. Bobo JK, Husten C. Sociocultural influences on smoking and drinking. Alcohol Res Health 2000; 24:225-32.

43. Goodman E, Huang B. Socioeconomic status, depressive symptoms and adolescent substance use. Arch Pediatr Adolesc Med 2002; 156:448-53.

44. Fergusson DM, Woodward LJ. Mental health, educational, and social role outcomes of adolescents with depression. Arch Gen Psychiatry 2002; 59:225-31.

45. Rohde P, Lewinsohn PM, Kahler CW, Seeley JR, Brown RA. Natural course of alcohol use disorders from adolescence to young adulthood. J Am Acad Child Adolesc Psychiatry 2001; 40:83-90.

Recibido el 02/Ago/2004

Versión final presentada el 29/Mar/2005 Aprobado el 18/Abr/2005 